Efraín Valencia Alanes. ${ }^{1}$ Dilean Elfy Ayaviri. ${ }^{2}$ Jimena Fernandez Zambrana. $^{3}$

Naicon Jean Camera Rech. $^{3}$

Alan F. Santos. ${ }^{3}$

Correspon dencia a:

${ }^{1}$ Coordinador de red cercado servicio departamental de salud.

Cochabamba - Bolivia

${ }^{2}$ Docente de Farmacología de la carrera de medicina UNITEPC.

Medico del centro de investigación de salud publica UNITEPC. CISPU

Cochabamba - Bolivia

${ }^{3}$ Centro de investigación medicina UNITEPC. CIMU

Cochabamba - Bolivia

efravaal@gmail.com dayaviri_med@unitepc. eso.bo

jimenafernandezzambrana@gmail.com

naiconcamera@gmail.com alanf_santos@uni-tepcmail.com

Procedencia y arbitraje: No comisionado, sometido a arbitraje externo

Recibido para publicación: 26 de diciembre del 2018 Aceptado para publicación: 27 de febrero del 2019

Citar como:

Re Ci Sa UNI

2019:6(1):15-19

\section{CARACTERÍSTICAS DE LA AUTOMEDICACIÓN EN EL DISTRITO 3 CIUDAD DE COCHABAMBA}

\section{THE AUTOMEDICATION CHARACTERISTICS IN COCHABAMBA CITY - DISTRICT 3 \\ CARACTERÍSTICAS DA AUTOMEDICAÇÃO NO DISTRITO 3 CIDADE DE COCHABAMBA}

\section{Resumen.}

Introducción: La automedicación es la principal causa de consumo de medicamentos dentro del uso irracional de fármacos, se ha convertido en un problema de salud, constituyendo un potencial de riesgo para las personas. El objetivo del estudio es, determinar características sobre la automedicación. Metodología: Es descriptivo, transversal y prospectivo. La muestra seleccionada corresponde a 1200 habitantes mayores de 18 años residentes del distrito 3 , ciudad de Cochabamba. Resultados: $36(3 \%)$ no consume medicamentos sin receta médica y $1164(97 \%)$ lo hace. Las fuentes de información para la automedicación son; Televisión $48(4 \%)$, internet $252(21 \%)$, familia $396(33 \%)$, prescripción anterior $324(27 \%)$ y Otras fuentes de información $180(15 \%)$. Los síntomas frecuentes para la automedicación son; Cefalea 372 (31\%), Mialgia 336 (28\%), Fiebre $288(24 \%)$, Diarrea $48(4 \%)$ y otros $156(13 \%)$. Los fármacos frecuentes que consumen por automedicación son; Analgésicos 348 (29\%), antibióticos 264 (22\%), Antigripales 216 (18\%), antinflamatorios $288(24 \%)$, Antiulcerosos $36(3 \%)$ y Vitaminas 48 (4\%). Discusión: La automedicación es elevado en relación a otros estudios, los familiares son la principal fuente de información para conocer sobre medicamentos y la cefalea es el síntoma principal para esta práctica con el consumo de analgésicos.

Palabras Clave: Automedicación. Impacto en la Salud. Población

\section{Summary.}

Introduction: Self-medication is the main cause of drug consumption within the irrational use of drugs, it has become a health problem, developing a potential risk for people. The objective of the study is to determine the characteristics about self-medication. Methodology: It is descriptive, transversal and prospective. The selected sample corresponds to 1200 habitants older than 18 years residents district 3 , in Cochabamba city . Results: $36(3 \%)$ do not consume drugs without a prescription and 1164 $(97 \%)$ do so. The sources of information for self-medication are; Television 48 (4\%), Internet $252(21 \%)$, family $396(33 \%)$, previous prescription $324(27 \%)$ and another information sources 
180 (15\%). The frequent symptoms for self-medication are; Headache 372 (31\%), Myalgia 336 (28\%), Fever 288 (24\%), Diarrhea 48 (4\%) and another 156 (13\%). The frequent drugs that they consume by self-medication are; Analgesics 348 (29\%), antibiotics 264 (22\%), Influenza 216 (18\%), anti-inflammatory 288 (24\%), Antiulcer agents $36(3 \%)$ and Vitamins 48 (4\%). Discussion: Self-medication is high in relation to other studies, the family is the main source of information to know about medications and headache is the main symptom for this practice of analgesics consumption.

Key words: Self Medication. Impacts on Health. Population.

Resumo.

Introdução: A automedicação é a principal causa do consumo de medicamentos dentro do uso irracional de medicamentos. Tornou-se um problema de saúde pública, constituindo um risco potencial para as pessoas. Objetivo do estudo é determinar características da automedicação. Metodologia: Descritiva, transversal e prospectiva. A amostra selecionada corresponde a 1.200 habitantes maiores de 18 anos residentes no distrito 3 , cidade de Cochabamba. Resultados: $36(3 \%)$ não consomem medicamentos sem receita médica e 1164 (97\%) o fazem. As fontes de informação para a automedicação são: televisão 48 (4\%), internet 252 (21\%), família 396 (33\%), prescrição prévia $324(27 \%)$ e outras fontes de informação 180 (15\%). Os sintomas frequentes para automedicação são: cefaleia 372 (31\%), mialgia 336 (28\%), febre 288 (24\%), diarreia $48(4 \%)$ e outros $156(13 \%)$. Os medicamentos frequentes que eles consomem são: analgésicos 348 (29\%), antibióticos 264 (22\%), antigripal 216 (18\%), anti-inflamatórios 288 (24\%), antiúlcera 36 (3\%) e vitaminas 48 (4\%). Discussão: A automedicação é alta em relação a outros estudos. Os familiares são a principal fonte de informação para se saber sobre medicamentos, e, a dor de cabeça é o principal sintoma dessa prática com o consumo de analgésicos.

Palavras chaves: Automedicação. Impactos na Saúde. População

\section{INTRODUCCIÓN}

Históricamente el propio tratamiento de los signos y síntomas de cualquier enfermedad que las personas padecen, ha sido la forma más utilizada para el mantenimiento de la salud. En el ámbito familiar, comunitario y cultural, ha sido la fuente de conocimiento de medicamentos existentes en cada entorno cultural, traspasando verbalmente dichos conocimientos a las sucesivas generaciones (1).

En los primeros documentos escritos, se recogía la existencia de un "experto" en la sanación, incluso se sabe que antiguamente se utilizaban partes de animales y plantas para medicarse (1).

La automedicación es una práctica, mediante el cual la persona recibe medicamentos por su propia iniciativa, por sugerencia de terceros con conocimiento empírico o por un consejo del personal de farmacia. Se ha practicado desde épocas ancestrales, y siempre fue impulsada por el deseo de las personas de combatir a las enfermedades que aquejaban a la sociedad y provocaban la muerte (2).

En el marco de la Declaración conjunta por la Federación Farmacéutica Internacional (FIP) y la Industria Mundial de la Automedicación Responsable. Surge la definición de automedicación como "el uso de medicamentos, sin receta, por iniciativa propia de las personas" (3). 
La automedicación implica el uso de medicamentos por parte del consumidor para tratar signos y síntomas (4).

Según Oruetaa, (5). La automedicación se define como la obtención y utilización de medicamentos, tanto de "prescripción con receta" como "sin receta", por parte de las personas, sin participación de un médico en el diagnóstico de la enfermedad.

De un modo general, la automedicación se entiende como la conducta que implica el tomar medicamentos según la propia iniciativa de la persona, o puede ser por consejo del personal de farmacia o de cualquier persona que no sea de la formación médica, de este modo se entiende que es sin la participación de un médico certificado, sin el auxilio de un diagnóstico y consentimiento con prescripción legal del medicamento.

Como agravantes de este escenario, además de las cuestiones culturales y sociales de la población, también existe la presión de industrias farmacéuticas que imponen a través de la publicidad, estas que induce el uso de los medicamentos como sinónimo de salud. Tal conducta de las industrias crea un problema grave para la salud pública y para la sociedad, que se denomina "medicalización de la salud" (6).

La facilidad del uso y de la libre disposición de los medicamentos de venta libre puede provocar no sólo errores en su aplicación sino también en el abordaje global de la enfermedad a la cual van dirigidos. (7)

Las personas no solo disponen de medicamentos de venta libre en el mercado, sino también aquellos están en venta bajo prescripción médica estricta. Esta conducta lleva al uso irracional de los medicamentos, siendo sobre todo los medicamentos más usados como analgésicos, antibióticos, antihistamínicos, antiácidos, antiinflamatorios y anticonceptivos de uso sistémico (7).

El objetivo del presente estudio es, determinar características sobre la automedicación en el distrito 3 ciudad de Cochabamba Bolivia 2018.

\section{METODOLOGÍA}

El tipo de investigación es descriptivo, transversal y prospectivo. La muestra seleccionada corresponde a 1200 habitantes mayores de 18 años residentes del distrito 3, ciudad de Cochabamba - Bolivia durante el año 2018.

Se hizo una encuesta como técnica de recolección de datos con la aplicación del instrumento de cuestionario. Así se buscó contemplar y aclarar los objetivos ya presentados de esta investigación.

\section{RESULTADOS}

Las 1200 encuestas mostraron los siguientes resultados; 36 (3\%) no consume medicamentos sin receta médica y 1164 (97\%) lo hace. Ver cuadro 1

Cuadro 1. Consumo de medicamentos sin receta médica en el distrito 3 ciudad de Cochabamba - Bolivia 2018.

\begin{tabular}{|l|c|c|c|c|}
\hline & SI & $\%$ & NO & $\%$ \\
\hline Consumen medicamentos sin receta medica & 36 & 3 & 1164 & 97 \\
\hline
\end{tabular}

Fuente: Propia de los autores

En relación a las fuentes de información para la automedicación los resultados mostraron; Televisión 48 (4\%), internet 252 (21\%), familia 396 (33\%), prescripción anterior 
$324(27 \%)$ y Otras fuentes de información 180 (15\%). Ver cuadro 2.

Cuadro 2. Fuentes de información para la automedicación en el distrito 3 ciudad de Cochabamba - Bolivia 2018

\begin{tabular}{|l|c|c|}
\hline Fuente de información & Numero & $\%$ \\
\hline Televisión & 48 & 4 \\
\hline Internet & 252 & 21 \\
\hline Familia & 396 & 33 \\
\hline Prescripción anterior & 324 & 27 \\
\hline Otro & 180 & 15 \\
\hline
\end{tabular}

Fuente: Propia de los autores

Los síntomas frecuentes para la automedicación son; Cefalea 372 (31\%), Mialgia 336 (28\%), Fiebre $288(24 \%)$, Diarrea $48(4 \%)$ y otros $156(13 \%)$. Ver cuadro 3

Cuadro 3. Síntomas frecuentes para automedicación en el distrito 3 ciudad de Cochabamba - Bolivia 2018

\begin{tabular}{|l|r|r|}
\hline Síntomas & Numero & Porcentaje \\
\hline Cefalea & 372 & 31 \\
\hline Mialgia & 336 & 28 \\
\hline Fiebre & 288 & 24 \\
\hline Diarrea & 48 & 4 \\
\hline Otros & 156 & 13 \\
\hline
\end{tabular}

Fuente: Propia de los autores

Los fármacos frecuentes que consumen por automedicación son; Analgésicos 348 (29\%), antibióticos 264 (22\%), Antigripales 216 (18\%), antinflamatorios 288 (24\%), Antiulcerosos 36 (3\%) y Vitaminas 48 (4\%). Ver cuadro 4.

Cuadro 4. fármacos frecuentes que consumen por automedicación en el distrito 3 ciudad de Cochabamba - Bolivia 2018

\begin{tabular}{|l|c|c|}
\hline Fármaco & Numero & Porcentaje \\
\hline analgésicos & 348 & 29 \\
\hline antibióticos & 264 & 22 \\
\hline Antigripales & 216 & 18 \\
\hline antinflamatorios & 288 & 24 \\
\hline Antiulcerosos & 36 & 3 \\
\hline Vitaminas & 48 & 4 \\
\hline
\end{tabular}

Fuente: Propia de los autores

\section{DISCUSIÓN}

La prevalencia de la automedicación fue del $97 \%$ porcentaje que se encuentra elevado en comparación con otros estudios similares, como el estudio realizado en la ciudad de Coronel Oviedo de Paraguay donde los resultados mostraron $48.3 \%$ (8) y otro realizado en Bogotá-Colombia dónde la prevalencia fue del 27,3\% (9)

Las fuentes de información frecuentes para obtener información de los medicamentos para la automedicación fueron; la familia en un 33\%, prescripción anterior $27 \%$ e internet $21 \%$, estos datos difieren en relación a otros estudios como por ejemplo de 
Cartagena Colombia que indica, familia 56,5\%, experiencias con prescripción anterior $2.3 \%$ e internet $1.2 \%$ (10)

La cefalea es la principal causa para la automedicación $31 \%$ y los analgésicos el principal medicamento consumido estos datos están relacionados con otros estudios similares (8).

Al finalizar la investigación llegamos a las siguientes conclusiones: La automedicación en el distrito 3 de la ciudad de Cochabamba Bolivia es elevado en relación a otros estudios, los familiares son la principal fuente de información para conocer sobre medicamentos y que la cefalea es el síntoma principal para esta práctica con el consumo de analgésicos.

\section{BIBLIOGRAFÍA}

1. Vicente VB. Estrategias para reducir los riesgos de la automedicación. Inf Ter Sist Nac Salud. 2000;24(6):147-52.

2. Regueira Betancourt SM, Rodríguez Fernández A, Hidalgo Fuentes K. Intervención sobre la automedicación en el Consultorio Médico de la Familia 130, año 2012. Revista Electrónica Dr. Zoilo E. Marinello Vidaurreta [revista en Internet]. 2015 [citado 2018 Oct 21];38(4): [aprox. 0 p.]. Disponible en: http://revzoilomarineIlo.sld.cu/index.php/zmv/article/view/587

3. database_file.pdf [Internet]. [citado 21 de Nov 2019]. Disponible en: https://www. fip.org/www/uploads/database_file.php?id=244\&table_id=

4. Ramos_rj.pdf [Internet]. [citado 21 de oct de 2018]. Disponible en: http://cybertesis.unmsm.edu.pe/bitstream/handle/cybertesis/3647/Ramos_rj.pdf;jsessioni$\mathrm{d}=835 \mathrm{~A} 999 \mathrm{AD} 459 \mathrm{E} 1 \mathrm{F0E8EB091B32E0C36D? \text {sequence=1 }}$

5. Orueta R, Toledano P, Gómez-Calcerrada R. Actualización en Medicina de Familia: Cumplimiento terapéutico. Med Fam-SEMERGEN. 2008;34(5):235-43.

6. Estudio.pdf [Internet]. [citado 21 de marzo de 2019]. Disponible en: http://odn.unne. edu.ar/Estudio.pdf

7. Lazcano L, Parra E, Umeres L, Valverde A. Causas que inducen la automedicación en estudiantes de primer y quinto curso de la Facultad de Medicina de la USFX. En: Participación ciudadana y desarrollo local, 2014, ISBN 978-476-5237-05-5, págs 53-62 [Internet]. ECORFAN; 2014 [citado 21 de Nov 2018]. p. 53-62. Disponible en: https://dialnet.unirioja.es/servlet/articulo?codigo $=4775745$

8. Escobar Salinas Jorge Sebastián, Ríos González Carlos Miguel. Prevalencia de automedicación en dos barrios de la ciudad de coronel Oviedo - Paraguay, marzo a mayo 2014. SCIENTIFICA [Internet]. 2014 [citado 2018 Nov 21]; 12(1): 46-50. Disponible en: http://www.revistasbolivianas.org.bo/scielo.php?script=sci_arttext\&pid=S1813-00542014000100008\&Ing=es.

9. López JJ, Dennis R, Moscoso SM. Estudio sobre la Automedicación en una Localidad de Bogotá. Rev Salud Pública. 1 de mayo de 2009;11(3):432-42.

10. Del Toro M, Díaz A, Barrios Z, Castillo IY. Automedicación y creencias en torno a su práctica en Cartagena, Colombia. Rev Cuid. 2017; 8(1): 1509-18 